\title{
Gene expression profiling reveals activation of the FA/BRCA pathway in advanced squamous cervical cancer with intrinsic resistance and therapy failure
}

Ovidiu Balacescu ${ }^{1 *}{ }^{*}$, Loredana Balacescu $^{1 \dagger}$, Oana Tudoran ${ }^{1}$, Nicolae Todor ${ }^{1}$, Meda Rus ${ }^{1}$, Rares Buiga ${ }^{1}$,

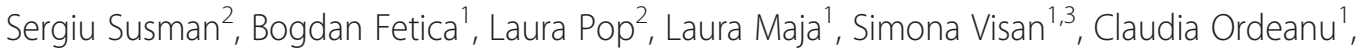
Ioana Berindan-Neagoe ${ }^{1,2^{*}}$ and Viorica Nagy ${ }^{1,2}$

\begin{abstract}
Background: Advanced squamous cervical cancer, one of the most commonly diagnosed cancers in women, still remains a major problem in oncology due to treatment failure and distant metastasis. Antitumor therapy failure is due to both intrinsic and acquired resistance; intrinsic resistance is often decisive for treatment response. In this study, we investigated the specific pathways and molecules responsible for baseline therapy failure in locally advanced squamous cervical cancer.
\end{abstract}

Methods: Twenty-one patients with locally advanced squamous cell carcinoma were enrolled in this study. Primary biopsies harvested prior to therapy were analyzed for whole human gene expression (Agilent) based on the patient's 6 months clinical response. Ingenuity Pathway Analysis was used to investigate the altered molecular function and canonical pathways between the responding and non-responding patients. The microarray results were validated by qRT-PCR and immunohistochemistry. An additional set of 24 formalin-fixed paraffin-embedded cervical cancer samples was used for independent validation of the proteins of interest.

Results: A 2859-gene signature was identified to distinguish between responder and non-responder patients. 'DNA Replication, Recombination and Repair' represented one of the most important mechanisms activated in non-responsive cervical tumors, and the 'Role of BRCA1 in DNA Damage Response' was predicted to be the most significantly altered canonical pathway involved in intrinsic resistance $(p=1.86 \mathrm{E}-04$, ratio $=0.262)$. Immunohistological staining confirmed increased expression of BRCA1, BRIP1, FANCD2 and RAD51 in non-responsive compared with responsive advanced squamous cervical cancer, both in the initial set of 21 cervical cancer samples and the second set of 24 samples.

Conclusions: Our findings suggest that FA/BRCA pathway plays an important role in treatment failure in advanced cervical cancer. The assessment of FANCD2, RAD51, BRCA1 and BRIP1 nuclear proteins could provide important information about the patients at risk for treatment failure.

Keywords: FANCD2, RAD51, BRCA1, BRIP1, Cervical cancer, Microarray, Treatment response

\footnotetext{
*Correspondence: obalacescu@yahoo.com; ioananeagoe29@gmail.com

${ }^{\dagger}$ Equal contributors

'The Oncology Institute "Prof Dr. Ion Chiricuta", 34-36 Republicii street, 400015 Cluj-Napoca, Romania

${ }^{2}$ Iuliu Hatieganu, University of Medicine and Pharmacy, 8 Babes street, 400012 Cluj-Napoca, Romania

Full list of author information is available at the end of the article
} 


\section{Background}

Cervical cancer, the third most commonly diagnosed cancer in women, with 529,800 cases in 2010 [1], represents a major problem in oncology due to treatment failure and distant metastasis. More than $85 \%$ of cervical cancers are diagnosed every year in developing countries, and approximately $90 \%$ of overall deaths occur in these countries. If detected at an early stage, cervical cancer represents one of the most successfully treated cancers. Unfortunately, because of the lack of screening programs in developing countries, cervical cancer is predominantly detected in advanced stages (IIB-IIIB). About half of the patients with advanced cervical cancer will develop recurrence or metastasis in the first 2 years after completion of therapy.

Although new anticancer drugs are constantly being developed, overcoming drug resistance is still a challenge. Therefore, there is an urgent need to identify new prognostic factors that could distinguish between patients with unfavorable prognoses from others with better prognoses.

Almost half of patients present baseline resistance (intrinsic resistance), and a large proportion of the remaining half will develop resistance during treatment (acquired resistance) [2]. Intrinsic resistance is often complex and occurs through several mechanisms, depending on the therapy regimen. The treatment for pre-invasive lesions is generally based on surgery; for invasive cervical cancers, the treatment is based on surgery and/or radiation and cisplatin-based chemotherapy [3]. The chemoradiotherapy treatment produces DNA double-strand breaks (DSBs), which is considered to be the most lethal form of DNA damage. DSBs are caused by radiation and platinum compounds based chemotherapy but also could be produced by endogenous damage, such as that caused by reactive oxygen species and collapsed replication forks. DNA damage induces a series of molecular responses that are responsible for the maintenance of genome integrity [4]. Deficiencies in DSB response and repair could represent important events for intrinsic resistance.

The diagnosis of baseline resistance in individual patients could improve the cancer treatment by the avoidance of inefficient therapy. Gene expression studies have been conducted across many tumor types to investigate the patterns of genes involved in intrinsic resistance. In cervical cancer, relatively few studies have been focused on identifying baseline resistance to chemoradiotherapy [5-7]. Therefore, the aim of our study was to investigate the specific pathways and molecules responsible for baseline therapy failure in locally advanced squamous cervical cancer.

\section{Methods}

\section{Sample collection}

Patient samples and clinical data with end points were obtained from the Departments of Radiotherapy and Pathology of The Oncology Institute 'Prof. Dr. I. Chiricuta', Cluj-Napoca, Romania. This study was approved by the ethics committee of The Oncology Institute 'Prof. Dr. Ion Chiricuta'. All patients gave informed consent in accordance with the Declaration of Helsinki.

Twenty-one patients with locally advanced squamous cell carcinoma (FIGO stage IIB-IIIB) were enrolled in the genomics study. A tissue fragment from a primary biopsy and a cervical lavage specimen were harvested from each patient prior to initiation of the therapy. Tissue samples were stored in liquid nitrogen until use for RNA extraction.

Corresponding formalin-fixed paraffin-embedded (FFPE) tissue samples were used for protein validation. Moreover, an additional set of 24 FFPE samples was used for independent immunohistochemistry validation of the data. All patients in the validation and study groups had the same including criteria. The clinical and histopathological characteristics of the patients included in this study are presented in Table 1.

\section{The therapy schedule}

The patients were treated with concomitant chemoradiotherapy (CRT) associated or not with surgery. The radiotherapy protocol includes external beam radiotherapy (EBRT) to the pelvis delivered by a linear accelerator at $15 \mathrm{MV}$ for a dose of $46 \mathrm{~Gy} / 23$ fractions and a cervical boost given by intracavitary high-dose-rate (HDR) brachytherapy (BT) in a dose of $10 \mathrm{~Gy} / 2$ fractions. Cisplatin was administered concomitant with the radiotherapy as a radiosensitizer. At this dose, patients were evaluated and,

Table 1 Baseline characteristics of the patients in the genomics study and IHC validation group

\begin{tabular}{lcc}
\hline Characteristics & $\begin{array}{c}\text { Genomics study } \\
\text { group }(\mathbf{n}=\mathbf{2 1})\end{array}$ & $\begin{array}{c}\text { IHC validation } \\
\text { group ( } \mathbf{n} \mathbf{2 4})\end{array}$ \\
\hline $\begin{array}{l}\text { Median age } \\
\text { (range), years }\end{array}$ & $46(27-73)$ & $52(28-62)$ \\
\hline $\begin{array}{l}\text { Median tumor } \\
\text { size (range), cm }\end{array}$ & $5(2-8)$ & $4(2-7)$ \\
\hline $\begin{array}{l}\text { Median hemoglobin } \\
\text { (range), g/dl }\end{array}$ & $12.7(7.9-14.4)$ & $13.3(10.2-14.9)$ \\
\hline FIGO stage & 10 & 8 \\
\hline II B & 5 & 11 \\
\hline III A & 6 & 5 \\
\hline III B & & 19 \\
\hline HPV subtype & 16 & 3 \\
\hline HPV 16 & 3 & 2 \\
\hline Other high-risk* & 2 & 15 \\
\hline Negative & 9 & \\
\hline Treatment response & & \\
\hline CR & & \\
\hline NCR & & \\
\hline
\end{tabular}

*other high-risk in study group: 33,58,73.

other high-risk in validation group: $31,45,58$. 
according to tumor response, further of CRT (EBRT until 60 Gy concomitant with cisplatin and HDR BT until 20 Gy) or surgery (radical abdominal hysterectomy with pelvic lymphadenectomy) was decided. In our internal protocol, surgery was recommended, but not mandatory, being a patient's option. The tumor response was clinically evaluated at 6 months after the end of the CRT treatment and was defined as complete response (CR) or non-complete response (NCR) (partial response and stable disease). For the patients that underwent surgery, the histopathological evaluation confirmed the clinical response.

\section{RNA extraction and purification}

Tumor sections with a minimum of $70 \%$ tumor cells were harvested by macrodissection from primary biopsies of cervical cancers. Total RNA was extracted with TriReagent (Sigma-Aldrich) and purified using an RNeasy Mini kit (Qiagen) according to the manufacturer's protocols. Extracted RNA was assessed for quality with a Labon-a-chip Bioanalyzer 2100 (Agilent Technologies). The RNA Integrity Number (RIN) and rRNA 28S/18S ratio were used to define the quality of the total RNA. The RNAs with RINs $>7.5$ and rRNA $28 \mathrm{~S} / 18 \mathrm{~S}$ ratios $>1.8$ were used for further analysis. RNA concentrations were adjusted using a NanoDrop ND-1000 spectrophotometer (NanoDrop Technologies).

\section{HPV genotyping}

Genomic DNA was extracted from $1 \mathrm{ml}$ of cervical lavage using a High Pure DNA extraction kit (Roche). HPV genotypes, including 37 high- and low-risk genotypes, were identified with the Linear Array HPV Genotyping Test (Roche) according to the manufacturer's protocol.

\section{Oligonucleotide microarray technology}

Agilent oligonucleotide technology was used to measure gene expression changes in the samples of interest. Microarray probes (cRNA-Cy3) were synthesized from $200 \mathrm{ng}$ of total RNA in two reaction steps using a one-color Agilent Low Input Quick Amp Labeling Kit according to the manufacturer's instructions. All labeled cRNAs (Cy3) were purified using an RNeasy Mini kit (Qiagen) and were evaluated for quality control using a Nanodrop ND-1000 spectrophotometer. cRNAs with minimum yields of $1.65 \mu \mathrm{g}$ and specific activities of $6 \mathrm{pmol} / \mu \mathrm{l} \mathrm{Cy3}$ per $\mu \mathrm{g}$ cRNA were selected for further analysis. After fragmentation to an average size of 60 - 100 nucleotides, each cRNA was hybridized for 17 hours at $65^{\circ} \mathrm{C}$ to whole-human-genome $4 \times 44 \mathrm{~K}$ microarray slides (product G4112F; Agilent) following the manufacturer's protocol (Agilent Technologies). The slides were scanned with an Agilent G2505B US45102867 microarray scanner, and gridding was performed with Feature Extraction Software v.10.5.1.1.
The microarray data have been deposited in the NCBI Gene Expression Omnibus (GEO) repository under accession number GSE56363.

\section{Microarray data analysis}

The microarray data, including median foreground and background intensities, flags and feature annotations, were imported into R/Bioconductor. The association between $\log 2$ values of background and foreground intensities across each array was estimated by computing Pearson correlation coefficients. Suitable R packages (arrayQualityMetrics, limma, marray) were used for quality control, normalization, filtering and data summarization. Betweenarray normalization was performed using the quantile normalization method. The median normalized signals were used for further data analysis. To reduce the number of non-informative features, the probes with saturated and non-uniform signals present in more than $15 \%$ of the samples were removed. Differentially expressed genes/sequences between non-responder and responder samples were selected using the moderated $t$-statistic. This method is an improvement over the standard t-statistic, as it allows elimination of the influence of random small withingroup variance by sharing information across genes. The Benjamini and Hochberg method was used to adjust the p-values for multiple testing (adjusted p-value $<0.05$ ). Only genes/sequences with at least a 1.5-fold change in expression between the studied groups were considered differentially expressed. The hierarchical clustering using Euclidean distances and Ward method was further performed to cluster the similarities in expression between genes/samples.

\section{Functional analysis}

The dataset containing differentially expressed genes was uploaded into the Ingenuity Pathway Analysis (IPA) software (Ingenuity ${ }^{\oplus}$ Systems, http://www.ingenuity.com) and was associated with the biological functions and canonical pathways in the Ingenuity Knowledge Base. Fisher's exact test $(\mathrm{p}<0.05)$ was used to assess the significance of the associations between genes in the dataset and biological functions or canonical pathways. In addition, for canonical pathways, a ratio was computed between the number of molecules from the dataset and the total number of molecules in that pathway.

\section{Quantitative real-time PCR (qRT-PCR)}

The First Strand cDNA Synthesis Kit (Roche) was used to reverse transcribe $200 \mathrm{ng}$ of total RNA. Five microliters of 1:10 (v/v)-diluted cDNA was amplified in a final volume of $20 \mu \mathrm{l}$ using a LightCycler 480 (Roche). The amplification was performed with $1 \mu \mathrm{M}$ specific primers (Tib Molbiol) and a $0.2 \mu \mathrm{M}$ specific hydrolysis probe from the Universal Probe Library (UPL). The primers and UPL probes were designed with Roche Applied Science software as follows: 
BRCA1 (NM_007294.3): F-ttgttgatgtggaggagcaa, R-ttgttgat gtggaggagcaa (UPL\#11); BRCA2 (NM_000059.3): F-agctta ctccggccaaaaa, R-ttcctccaatgcttggtaaataa (UPL\#50); RAD51 (NM_001164269.1): F-tgagggtacctttaggccaga, R-cactgccaga gagaccatacc (UPL\#66); FANCD2 (NM_033084.3): F-cgacttg accaaacttcct, R-tcctccaatctaatagacgacaact (UPL\#9); BRIP1 (NM_032043.1): F-aatggcacttcatcaacttgtc, R-tggatgcctgtttc ttagca (UPL\#71); BLM (NM_000057.2): F-gatcagaaagcacca cccata, R-tcagccatggtgtcacattc (UPL\#34); and 18S rRNA (NR_003286.2): F-gcaattattcccatgaacg, R- gggacttaatcaacgcacgc (UPL\#48). Thermal cycling conditions were set as follows: activation at $95^{\circ} \mathrm{C}$ for 10 minutes; followed by 40 cycles of amplification, including denaturation at $95^{\circ} \mathrm{C}$ for 15 seconds, annealing at $55^{\circ} \mathrm{C}$ for 20 seconds and extension at $72^{\circ} \mathrm{C}$ for 1 second; followed by a cooling step at $40^{\circ} \mathrm{C}$ for 30 seconds. The relative expression levels of target genes (NCR vs. CR) were calculated using the $\Delta \Delta \mathrm{Ct}$ method [8] after normalizing to $18 \mathrm{~S}$ housekeeping gene.

\section{Immunohistochemistry (IHC)}

Immunohistochemistry was performed on FFPE 4- $\mu$ m thick tissue sections, using a standard protocol. Following deparaffinization and rehydration of the tissue sections, antigen retrieval was performed for 20 minutes in $0.01 \mathrm{M}$ citrate buffer ( $\mathrm{pH}$ 6.0) using the boiling process (pressure cooker). Endogenous peroxidase was blocked with $\mathrm{H}_{2} \mathrm{O}_{2}$ (3\%). Blocking of the nonspecific reactions was performed using the Novocastra Protein block $^{\mathrm{TM}}$ solution. The sections were incubated 30 minutes with primary antibodies at room temperature in a humid chamber. The immunohistochemical staining was performed using the following dilutions for the primary monoclonal antibodies: 1:400 for BRCA1 (BioVision Inc., OH, USA, clone\#3364-100), 1:200 for BRCA2 (Covalab, Cambridge, UK, clone pab0457-0), 1:200 for FANCD2 (Thermo Pierce Biotechnology Inc., IL, USA, clone PA1-16548), 1:20 for Rad51 (Thermo Pierce Biotechnology Inc., IL, USA, clone MA5-14416) and 1:300 for BPRIP1 (Abcam, Cambridge, UK, product number ab151509). Sections were sensitized using Post Primary Block ${ }^{\mathrm{TM}}$, and then incubated with NovoLink ${ }^{\mathrm{Tm}}$ polymer containing the secondary antibody. The peroxidase reaction was developed using diamino-benzidine tetrachloride (DAB) as chromogen. Sections were counterstained with hematoxylin.

The IHC staining was automatically assessed using the ImmunoRatio free web-based application [9]. The application is conceived for automated image analysis of immunohistochemical nuclear staining like estrogen receptor (ER), progesterone receptor (PR), or Ki-67. Briefly, for every case 3 different representative images of immunostained sections were taken using a CX41 Olympus microscope coupled with a high resolution video camera AV5100M (MegaVideo IP camera, Arecont Vision). The application performs the segmentation of brown (DAB-colored), and hematoxylinstained nuclei, than calculates the labeling index as the percentage of brown stained nuclear area over the total nuclear area. The system also produces a pseudo-colored result image, illustrating the area segmentation. Every generated image was checked for consistency by two pathologists (BR and SS). Only the correct segmented images were accepted for further analysis.

\section{Statistical methods}

The follow-up endpoint for each patient represents a binary evaluation of the treatment response at 6 months after the end of the treatment. All existing factors were compared when examining the two groups of patients (CR and NCR).

Categorical factors were analyzed using a chi-squared test, and when reduced numbers of observations were present, we applied Yates' correction [10]. A comparison of medians was performed using the median test and two-tailed unpaired $t$ test was used to evaluate for differences in gene expression between groups of interes ( $\mathrm{NCR}$ vs $\mathrm{CR}$ ). The strengths of the association between genes of interest as well as between PCR and microarray results were tested with a Pearson parametric test. The receiver operating characteristic (ROC) curve was used to evaluate the predictive accuracy of genes of interest in the differentiation between samples with or without complete remission. [11]. The calculation of the area under curve (AUC) and test equality with a value of 0.5 was performed according to Bamber and Hanley [12,13]. The point of optimal classification was considered the point nearest to $(0.1)$ of the absolute classification. Unpaired t-test on arcsine-transformed data was used to determine whether the proportion of stained nuclear protein was different between non-responders and responders samples, in both genomic and IHC validation groups.

All differences with $\mathrm{p}<0.05$ were considered statistically significant. The confidence intervals were evaluated with the level of significance equal to 0.05 .

\section{Results}

\section{Patient and tumor characteristics}

FIGO staging evaluation of the patients included in this study revealed that approximately $48 \%$ of the patients were in stage II, while the rest of $52 \%$ were in stage III. Among these, 2 patients tested negative for HPV, whereas HPV-16 subtype has been detected in the majority of the cases. Based on 6 months treatment outcome evaluation twelve patients presented complete remission and were assigned to the CR group, while the rest of 9 patients that partially responded or had stable disease were assigned to the NCR group. We observed higher median age value in the responders group $(\mathrm{p}<0.01)$, however prognostic factors such as tumor size, hemoglobin and FIGO stage were balanced between the NCR and CR groups (Table 2). Since almost all the patients presented HPV 16-positive tumors, the association between HPV subtype and treatment outcome could not be assessed. 
Table 2 Association between clinical and histopathological data and treatment response

\begin{tabular}{|c|c|c|c|c|}
\hline Characteristics & $\begin{array}{c}\text { No. of patients } \\
n(\%)\end{array}$ & $\begin{array}{c}\text { CR group } \\
n\end{array}$ & $\begin{array}{c}\text { NCR group } \\
n\end{array}$ & $p$ \\
\hline Study population & $21(100 \%)$ & 12 & 9 & \\
\hline \multicolumn{5}{|l|}{ Age (years) } \\
\hline Median & 21 & 56.5 & 42 & $<0.01$ \\
\hline Range & & $28-73$ & $27-55$ & \\
\hline \multicolumn{5}{|l|}{ Tumor size $(\mathrm{cm})$} \\
\hline Median & 21 & 4.75 & 5 & 0.054 \\
\hline Range & & $2.0-6.5$ & $2.5-8.0$ & \\
\hline \multicolumn{5}{|l|}{ Hemoglobin (g/dl) } \\
\hline Median & 21 & 12.75 & 12.2 & 0.8 \\
\hline Range & & 7.9-14.2 & $10.1-14.4$ & \\
\hline \multicolumn{5}{|l|}{ FIGO stage } \\
\hline$\| B$ & $10(47.62 \%)$ & 4 & 6 & 0.62 \\
\hline IIIA & $5(23.81 \%)$ & 4 & 1 & \\
\hline$\| \mathrm{IIB}$ & $6(28.57 \%)$ & 4 & 2 & \\
\hline \multicolumn{5}{|l|}{ HPV subtype } \\
\hline 16 & 16 (76.19\%) & 10 & 6 & - \\
\hline Other high-risk & $3(14.29 \%)$ & 1 & 2 & \\
\hline Negative & $2(9.52 \%)$ & 1 & 1 & \\
\hline
\end{tabular}

\section{Gene expression profiling of cervical cancer samples}

Gene expression profiles for NCR and CR samples were generated using one-color hybridization to whole human genome arrays carrying 43,376 biological sequences. We assessed the quality of the array before and after normalization and we did not detect batch effects or outlier arrays. We observed a weak correlation between background and foreground intensities across each array ( $\mathrm{r}$ range, 0.06 to 0.2 ), therefore we did not perform background correction. To improve data quality, a filtering step was applied. A total number of 40,998 sequences passed the filtering criteria and were used for further analysis. In class comparison analysis we identified a signature of 2859 genes whose differential expression in non-responder compared to responder samples exceeded 1.5-fold at an adjusted p-value $<0.05$. Of these, 1501 genes were up-regulated and 1358 genes were down-regulated in NCR compared with CR.

To highlight the differences in gene expression a supervised hierarchical clustering was performed on the set of differentially expressed genes. Based on expression profiles, non-responder and responder samples were grouped in two distinct main clusters (Figure 1).

\section{Functional profile assessment}

To obtain a global view of the altered biological functions and canonical pathways that could be responsible for intrinsic resistance in cervical cancer, we performed functional analysis in IPA. We chose to evaluate the biological functions and canonical pathways because it provides more robust results than studying individual genes. Sixty-five significant molecular functions have been predicted in IPA $(p<0.05)$ to be mediated by differentially expressed genes identified in NCR vs. CR samples. 'Cellular Movement' $(\mathrm{p}=5.30 \mathrm{E}-08-1.22 \mathrm{E}-02)$ was the top biological function mediated by these genes followed by 'Cell Cycle' ( $p=$ 7.12E-07-1.22E-02) and 'DNA Replication, Recombination and Repair' ( $\mathrm{p}=7.12 \mathrm{E}-07-1.18 \mathrm{E}-02)$. The dataset of differentially expressed genes were also integrated in 34 canonical pathways. The 'Role of BRCA1 in DNA Damage Response' was predicted to be the most significantly activated canonical pathway $(\mathrm{p}=1.86 \mathrm{E}-04)$, which suggests a baseline intrinsic resistance of non-responding cervical cancer tumors. The top five molecular and cellular functions and the canonical pathways with associated p-values are presented in Table 3.

It is known that cancer becomes resistant to therapy by restoring the DNA repair machinery; therefore, we focused our attention on the genes involved in 'DNA Replication, Recombination and Repair' molecular mechanisms. In total, 124 genes from our dataset were listed in these mechanisms (Additional file 1). The vast majority of genes $(\mathrm{n}=$ 92) were overexpressed with fold change between 1.503 and 2.867 while 32 genes were down-regulated (fold change: -10.471 to -1.509 ) in NCR vs. CR cervical samples. Among these genes, seventeen (RAD51, BRIP1, BLM, BRCA1, BRCA2, BRCC3, HLTF, FANCD2, FANCI, FANCM, FANCL, ATF1, E2F4, E2F2, SMARCA2, SMARCA4 and RFC1) were significantly associated in IPA with the 'Role of BRCA1 in DNA Damage Response' pathway $(\mathrm{p}=1.86 \mathrm{E}-04$, ratio $=0.262)($ Table 4$)$. The overexpression of BRCA1, BRCA2, RAD51, BRIP1 (BACH1), FANCD2, BLM and RFC in non-responding versus responding cervical cancer samples suggests that DNA repair mechanism activation occurs through cell cycle arrest and homologous recombination (Figure 2).

\section{qRT-PCR validation of the microarray results}

In order to assess the accuracy of microarray results, six genes including RAD51, BRIP1 (BACH1), BRCA1, BRCA2, BLM and FANCD2 involved in the 'Role of BRCA1 in DNA Damage Response' pathway were selected for validation by qRT-PCR. The fold changes calculated between NCR vs. CR samples revealed at least 3 -fold up-regulation for all genes of interest (Figure 3). We assessed the correlation between the qRT-PCR and microarray results by computing Pearson's correlation coefficients for each gene. A strong correlation between the two methods was observed $(\mathrm{r}=0.705-0.835)$ (Table 5). 


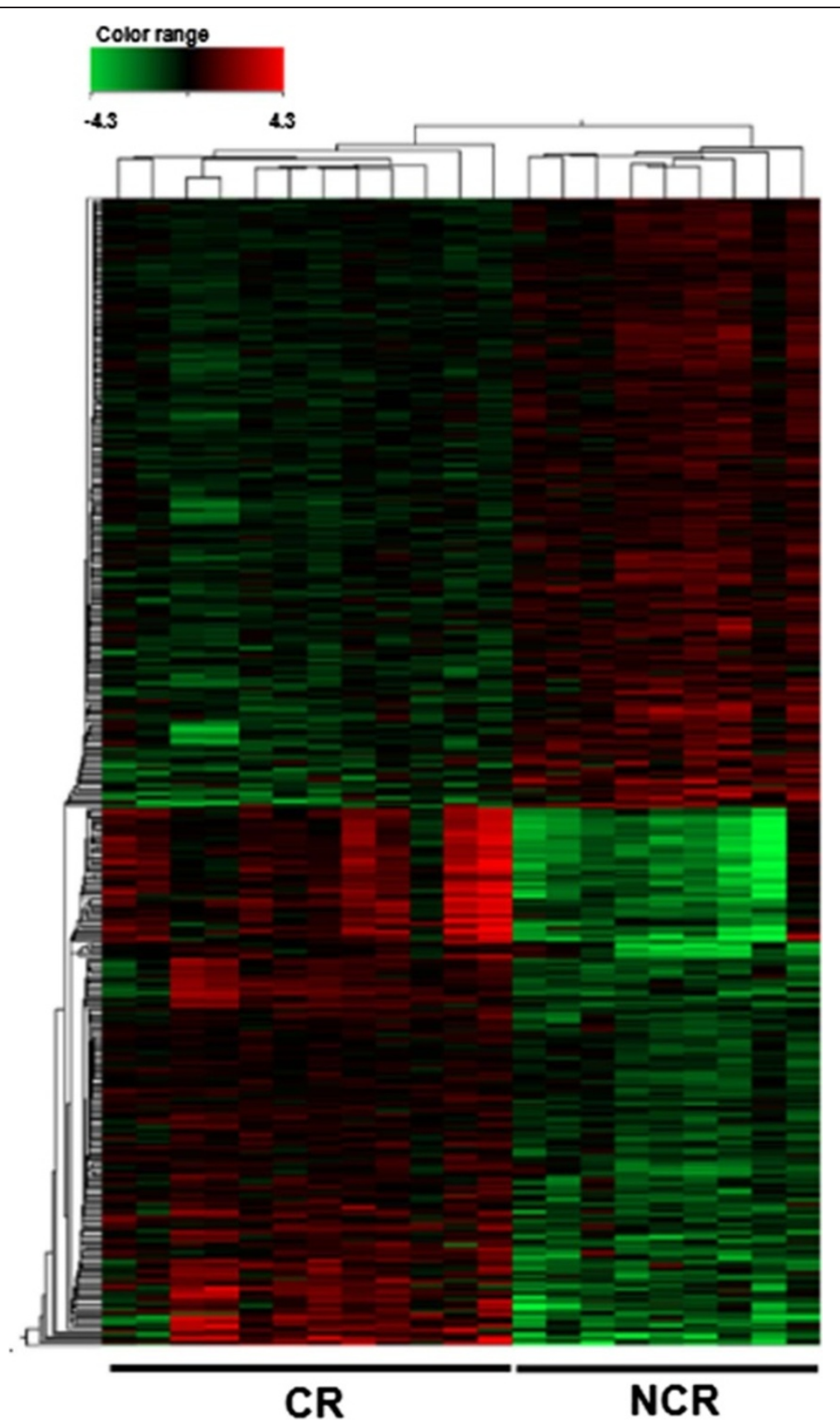

Figure 1 Heatmap of differentially expressed genes between CR $(n=9)$ and NCR $(n=12)$ samples obtained from supervised hierarchical clustering using Euclidean distances and Ward method. The color indicates the level of mRNA expression: red - higher level of expression, green - lower level of expression, black - no expression changes (each row represents a gene and each column represents a sample). The CR samples were clustered together and clearly separated from NCR samples.

Assessment of the prognostic significance of genes involved in 'Role of BRCA1 in DNA Damage Response' pathway

We estimated the prognostic significance of the six selected genes by the ROC analysis. We analyzed the ROC curves for all previously known potential factors, including age, tumor size, hemoglobin, along with our potential markers: BRCA1, BRCA2, RAD51, FANCD2, BLM and BRIP1. If the $p$-value was not significant $(p>0.05)$, then the AUC, sensitivity, specificity and optimal classification point were omitted. The investigated genes discriminated between the patients in the NCR and CR groups $(\mathrm{p}<0.01)$ suggesting a superior predictive value compared to classical factors such as tumor size and hemoglobin. The summary 
Table 3 The top significant molecular and cellular functions identified by IPA

\begin{tabular}{lll}
\hline Molecular and cellular functions & $\mathbf{p}$ value & No. genes \\
\hline Cellular movement & $5.30 \mathrm{E}-08-1.22 \mathrm{E}-02$ & 344 \\
\hline Cell cycle & $7.12 \mathrm{E}-07-1.22 \mathrm{E}-02$ & 233 \\
\hline $\begin{array}{l}\text { DNA replication, recombination } \\
\text { and Rrepair }\end{array}$ & $7.12 \mathrm{E}-07-1.18 \mathrm{E}-02$ & 124 \\
\hline Cellular development & $1.16 \mathrm{E}-06-1.22 \mathrm{E}-02$ & 433 \\
\hline $\begin{array}{l}\text { Cellular assembly } \\
\text { and organization }\end{array}$ & $4.97 \mathrm{E}-06-1.22 \mathrm{E}-02$ & 322 \\
\hline $\begin{array}{l}\text { Canonical pathways } \\
\begin{array}{l}\text { Role of BRCA1 in DNA } \\
\text { damage response }\end{array}\end{array}$ & $\mathbf{p}$ value & Ratio \\
\hline $\begin{array}{l}\text { Primary immunodeficiency } \\
\text { signaling }\end{array}$ & $1.86 \mathrm{E}-04$ & $17 / 65(0.262)$ \\
\hline $\begin{array}{l}\text { G protein signaling } \\
\text { mediated by Tubby }\end{array}$ & $3.97 \mathrm{E}-03$ & $11 / 62(0.177)$ \\
\hline $\begin{array}{l}\text { Aryl hydrocarbon } \\
\text { Rreceptor signaling }\end{array}$ & $5.05 \mathrm{E}-03$ & $9 / 42(0.214)$ \\
\hline $\begin{array}{l}\text { Regulation of actin-based } \\
\text { motility by Rho }\end{array}$ & $5.39 \mathrm{E}-03$ & $25 / 161(0.155)$ \\
\hline
\end{tabular}

of the ROC curves (AUC, specificity and sensitivity) for all six genes is presented in Table 6 .

The correlations between the target genes BRCA1, BRCA2, RAD51, FANCD2, BLM and BRIP1 indicated that all genes were highly correlated with each other. The correlation coefficients were between 0.69 (BRCA2 vs. BRIP1) and 0.93 (BRCA1 vs. BRIP1) (Figure 4).

\section{IHC validation of the microarray results}

Immunohistochemical staining was performed to obtain further validation of microarray findings. We assessed the protein expression of RAD51, BRIP1 (BACH1), BRCA1, BRCA2, BLM and FANCD2 in all 21 samples used in the genomic study (Figure 5). For BLM gene we did not identified a specific monoclonal antibody (MoAb), therefore this gene could not be taken into account for protein validation. An average percentage of nuclear staining on 3 different representative images of every sample was calculated for every protein of interest. We observed a significantly increased protein levels of FANCD2, BRCA1, RAD51 and BRIP1 in the nuclei of the NCR compared to the CR cervical tumors. No difference was observed for nuclear protein expression of BRCA2 in NCR compared to $\mathrm{CR}$ tissues. A ratio between nuclear protein expressions in NCR and CR groups was calculated (Table 7).

An additional set of 24 FFPE squamous cervical samples (15 CR and 9 NCR) was used as an independent validation of the protein data. Increased protein levels of FANCD2, RAD51, BRCA1, and BRIP 1 (BACH1) in NCR compared to $\mathrm{CR}$ cervical tumors groups were confirmed on the validation set (Table 7).

\section{Discussion}

Cervical cancer continues to represent a major health problem for women from developing countries. Cervical cancer lethality occurs because most patients are first diagnosed in advanced stages. Even if early stages are successfully treated,

Table 4 Genes involved in the "Role of BRCA1 in DNA Damage Response" pathway with associated p-values obtained from microarray experiment

\begin{tabular}{lcccc}
\hline Ref seq & Gene symbol & Fold regulation (NCR vs CR) & Adjusted p-value & Description \\
\hline NM_005171 & ATF1 & 1,747 & 0,013 & activating transcription factor 1 \\
\hline NM_000057 & BLM & 2,430 & 0,030 & Bloom syndrome, RecQ helicase-like \\
\hline NM_007300 & BRCA1 & 2,225 & 0,008 & breast cancer 1, early onset \\
\hline NM_000059 & BRCA2 & 1,842 & 0,011 & breast cancer 2, early onset \\
\hline NM_001018055 & BRCC3 & 1,621 & 0,016 & BRCA1/BRCA2-containing complex, subunit 3 \\
\hline NM_032043 & BRIP1 & 2,353 & 0,018 & BRCA1 interacting protein C-terminal helicase 1 \\
\hline NM_004091 & E2F2 & 2,137 & 0,048 & E2F transcription factor 2 \\
\hline NM_001950 & E2F4 & $-1,791$ & 0,013 & E2F transcription factor 4, p107/p130-binding \\
\hline NM_001018113 & FANCB & 2,216 & 0,010 & Fanconi anemia, complementation group B \\
\hline NM_033084 & FANCD2 & 1,613 & 0,012 & Fanconi anemia, complementation group D2 \\
\hline NM_018062 & FANCL & 1,701 & Fanconi anemia, complementation group L \\
\hline NM_020937 & FANCM & 1,923 & 0,031 & Fanconi anemia, complementation group M \\
\hline NM_139048 & HLTF & 2,245 & 0,005 & helicase-like transcription factor \\
\hline NM_002875 & RAD51 & 2,767 & 0,010 & RAD51 homolog (S. cerevisiae) \\
\hline NM_002913 & RFC1 & 1,723 & 0,004 & replication factor C (activator 1) 1, 145 kDa \\
\hline NM_139045 & SMARCA2 & 1,659 & 0,041 & SWI/SNF related, matrix associated, actin dependent \\
& & regulator of chromatin, subfamily a, member 2 & SWI/SNF related, matrix associated, actin dependent \\
\hline NM_003072 & SMARCA4 & regulator of chromatin, subfamily a, member 4 \\
\hline
\end{tabular}




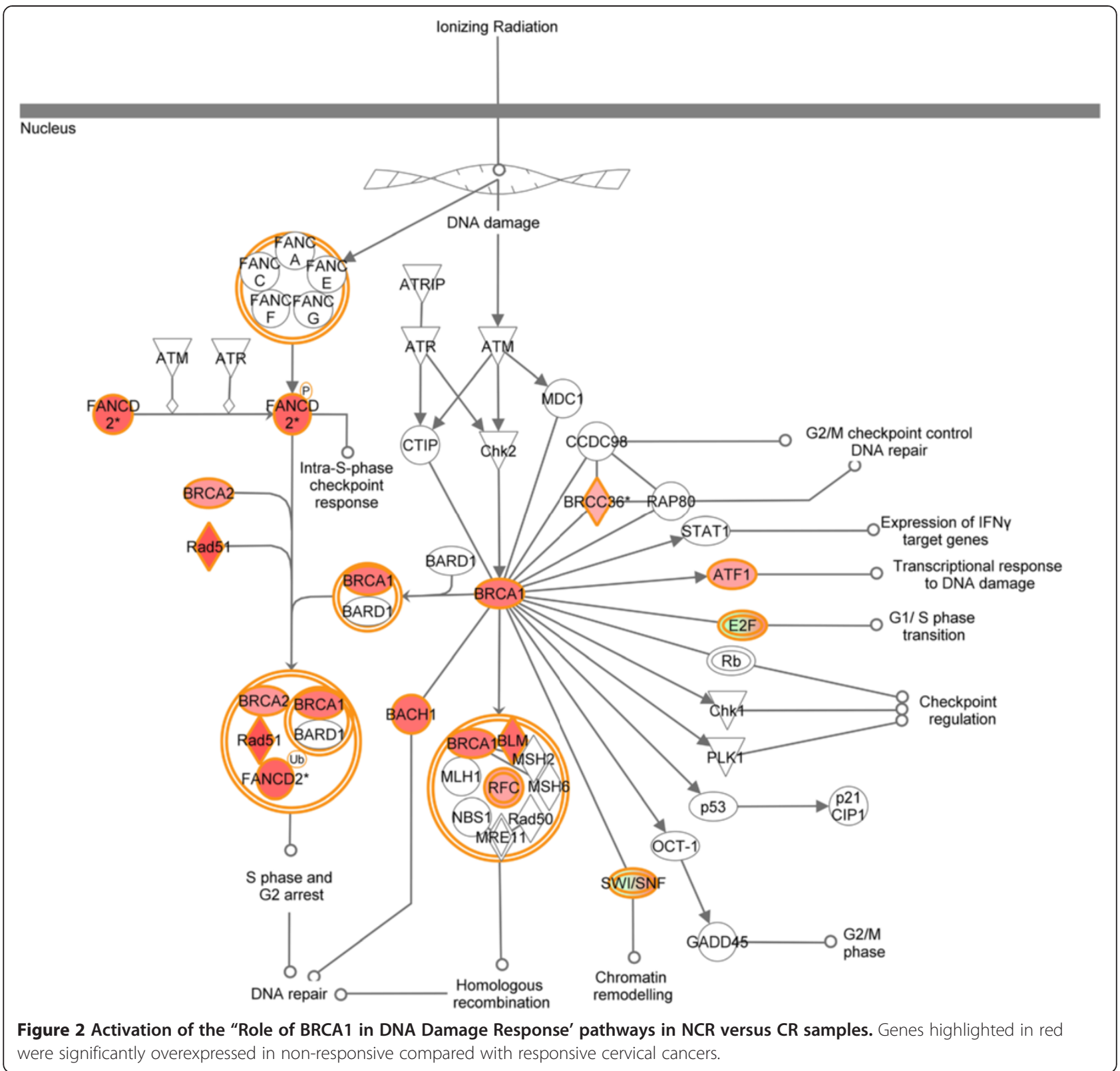

advanced cervical cancer represents a major problem due to increased rates of recurrence and distant metastasis. Although knowledge about tumor biology and various mechanisms of resistance has increased in recent years, different schedules of treatment, including new anticancer drugs, have not efficiently reduced the occurrence of drug resistance. Intrinsic resistance is often decisive for treatment failure; almost half of patients present with baseline resistance, rendering classical therapies ineffective.

In an effort to elucidate the patterns of genes involved in baseline resistance, we performed a genome-wide microarray assay on primary biopsies from patients with advanced cervical cancers with known clinical and histological responses. All of the patients included in the study received radiotherapy as the main therapy and cisplatin as a radiosensitizer Based on the microarray analysis, we identified a supervised gene expression profile that differed dramatically between the non-responding and responding cervical tumors. 'DNA Replication, Recombination and Repair' represents one of the most important molecular patterns identified as important for intrinsic resistance in cervical cancer. In our study, the non-responding cervical tumor cells had more active DNA damage repair machinery than responding cervical tumor cells, even before starting the therapy. In total, 92 out of the 124 identified genes involved in 'DNA replication, recombination and repair' were overexpressed in the non-responding tumors compared with the responding tumors (Additional file 1). 


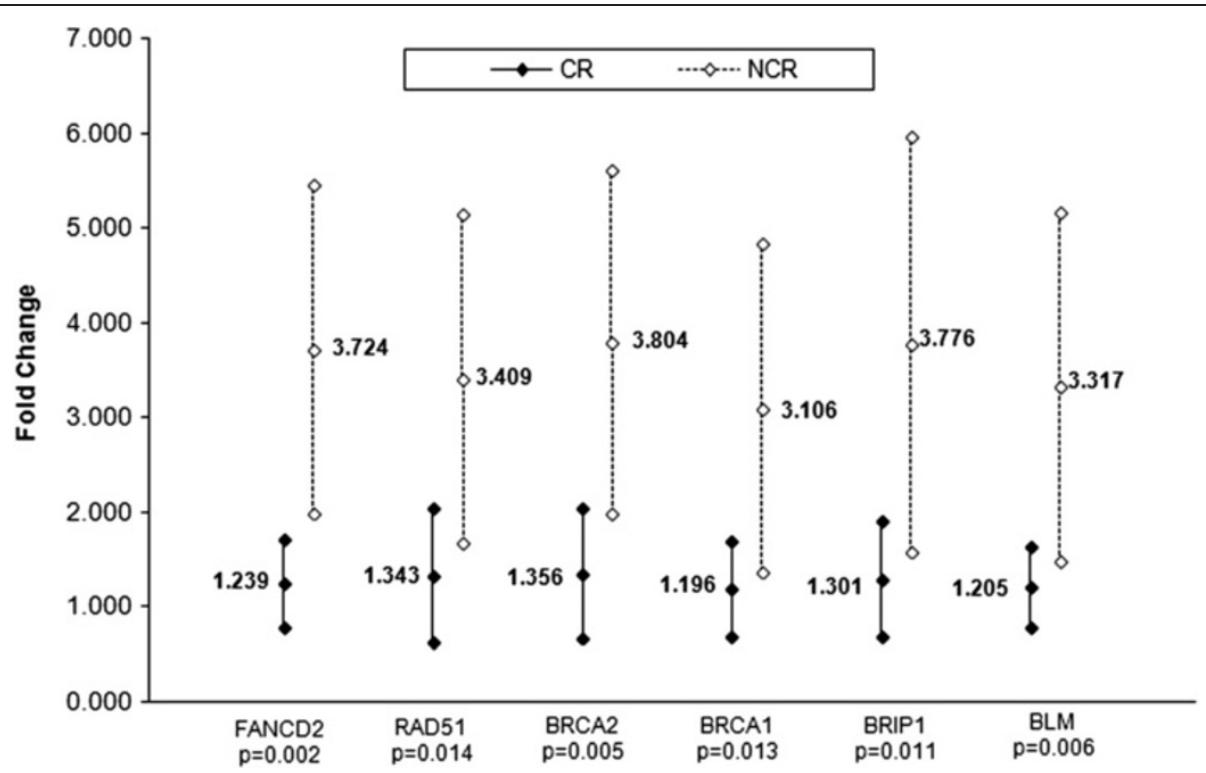

Figure 3 qRT-PCR validation data for six genes (FANCD2, RAD51, BRCA2, BRCA1, BRIP1/BCH1 and BML) involved in the 'Role of BRCA1 in DNA Damage Response' pathway. Fold change was calculated using the $\Delta \Delta C$ t method relative to the CR group.

Cancer cells become resistant to therapy by restoring DNA repair genes; therefore, we looked for pathways involved in the maintenance of DNA stability. By classifying the genes according to functional pathways, we identified the 'Role of BRCA1 in DNA Damage Response' as the most important canonical pathway involved in DNA repair (Table 3). To our knowledge, there are no studies that describe 'Role of BRCA1 in DNA Damage Response' pathway as predictive for treatment outcome in cervical cancer, even though a conserved pathway for increased DNA repair mediated by BRCA1 was described for other pathologies $[14,15]$. Among the genes significantly up-regulated in the BRCA1 canonical pathway, we focused our attention on a set of six genes that were considered of particular interest: BRCA1, BRCA2, RAD51, FANCD2, BACH1/ BRIP1/FANCJ and BLM. The expression of these genes detected by microarray was confirmed by qRT-PCR with good correlation (Table 5).

Early studies on BRCA1 and BRCA2 have reveled that both proteins are involved in DSB repair. In this study,

Table 5 Pearson's correlation coefficients of $\log _{2}$ fold change values obtained from microarray and PCR experiments

\begin{tabular}{lcc}
\hline Gene & Pearson coefficient & $\mathbf{p}$ \\
\hline BLM & 0.835 & $<0.0001$ \\
\hline BRIP1 & 0.811 & $<0.0001$ \\
\hline BRCA1 & 0.765 & $<0.0001$ \\
\hline BRCA2 & 0.721 & 0.0002 \\
\hline RAD51 & 0.705 & 0.0005 \\
\hline FANCD2 & 0.759 & $<0.0001$ \\
\hline
\end{tabular}

we showed that BRCA1 and BRCA2 overexpression in patients with advanced cervical cancer is associated with treatment failure. Several studies have pointed out that BRCA-deficient cells are inefficient at repairing DNA damage by homologous recombination (HR) $[16,17]$ and are thus more sensitive to chemotherapeutic drugs. Zhang et al. [18] reported that the E6 and E7 HPV oncoproteins interact with BRCA1 and alter its activity in cervical cancer cells. However, the association between high-risk HPV genotypes and treatment failure could not be evaluated in our study as our sample set did not comprise a sufficient number of other high-risk types. Recently, a so-called BRCAness gene expression profile has also been correlated with response to chemotherapy and outcome in patients with epithelial ovarian cancer [19]. BRCA1 is a component of the BASC complex that is important for efficient DNA

Table 6 ROC analysis for prognostic factors

\begin{tabular}{lcccccc}
\hline Nr.crt. & Variable & AUC & $\begin{array}{c}\text { Classification } \\
\text { point }\end{array}$ & Sensitivity & Specificity & p \\
\hline 1. & BRCA1 & 0.81 & $<0.858565$ & 0.92 & 0.78 & $<0.01$ \\
\hline 2. & BRCA2 & 0.86 & $<0.602903$ & 0.75 & 0.89 & $<0.01$ \\
\hline 3. & RAD51 & 0.81 & $<0.895025$ & 0.91 & 0.78 & $<0.01$ \\
\hline 4. & FANCD2 & 0.84 & $<0.673616$ & 0.83 & 0.78 & $<0.01$ \\
\hline 5. & BLM & 0.81 & $>0.871154$ & 0.63 & 0.99 & $<0.01$ \\
\hline 6. & BRIP1 & 0.81 & $>0.606256$ & 0.78 & 0.75 & $<0.01$ \\
\hline 7. & Age & 0.86 & $>46$ & 0.75 & 0.89 & $<0.01$ \\
& (years) & & & & & \\
\hline 8. & Tumor & 0.65 & - & - & - & 0.11 \\
& size (cm) & & & & & $(\mathrm{NS})$ \\
\hline 9. & Hb (g/dl) & 0.64 & - & - & - & 0.39 \\
& & & & & & (NS) \\
\hline
\end{tabular}



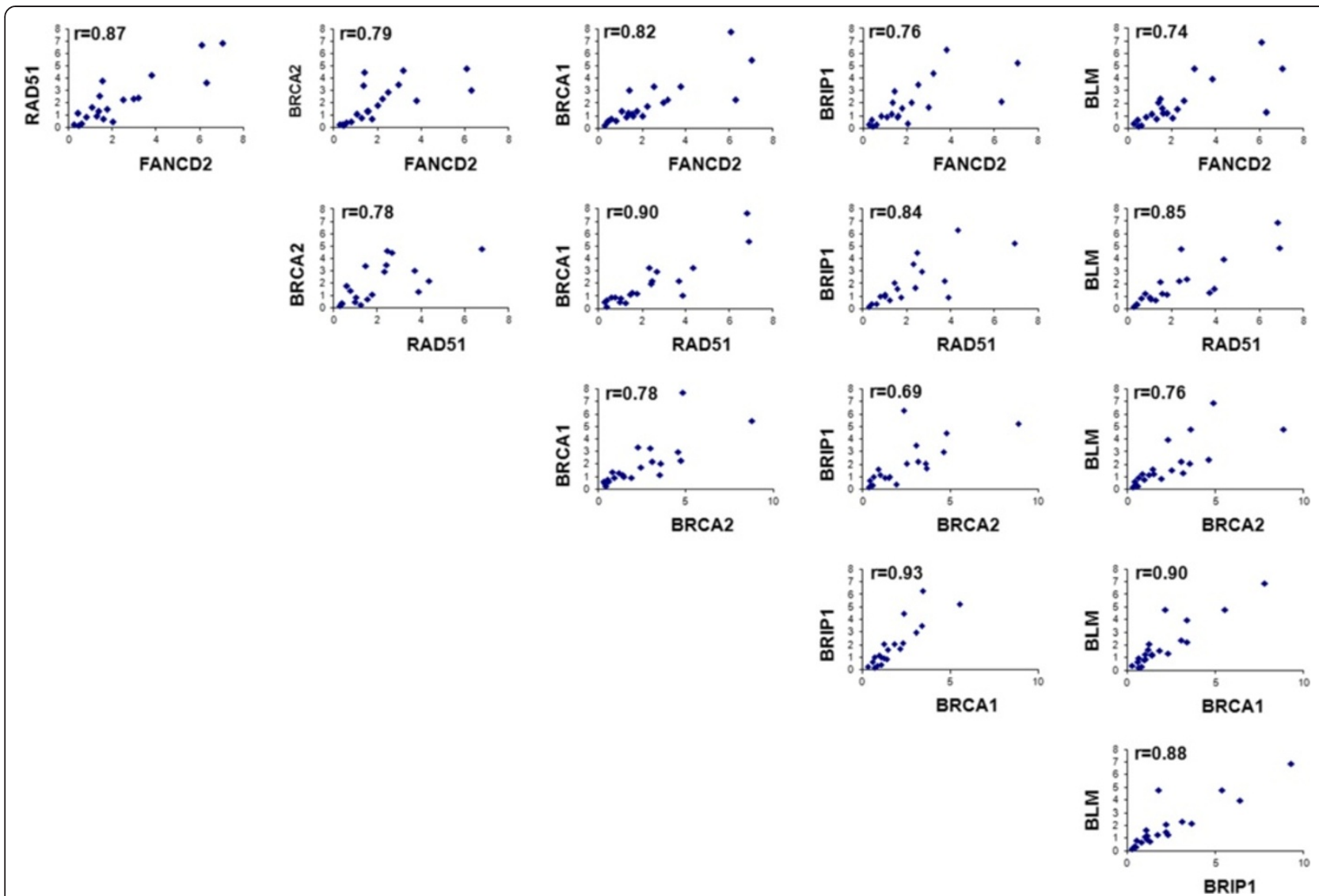

Figure 4 Pearson correlations between fold change values of the target genes.

repair. MSH2/MSH6, PMS2/MLH1, BLM helicase and the replication factor $\mathrm{C}$ (RFC) represent other important members of the BASC complex [20].

Our microarray data pointed out an increased level of BLM and RFC1 in the non-responding cervical cancers compared with the responding cancers. Additionally, BRCA1 associates with the SWI/SNF chromatin-remodeling complex and FANCD2 [21] and plays a role in regulating the cellular localization of BACH1/BRIP1 (BRCA1associated carboxyl-terminal helicase). BRCA2 is also involved in DNA repair; the protein interacts specifically with RAD51, an essential protein involved in HR [22]. In our efforts to understand the molecular basis of treatment response in advanced cervical cancer, besides the BRCA pathway, we also found the fanconi anemia (FA) complementation group, FANCD2, FANCL, FANCM, FANCJ/BRIP1/BACH and FANCI, to be involved in intrinsic resistance to chemo-radiotherapy. These FA proteins are closely related to the BRCA1 and BRCA2 gene products and their partner proteins and are required for cellular resistance to agents that cause DNA interstrand cross-links (ICLs) [23]. The FANCD2 protein colocalizes to nuclear foci together with BRCA1, BRCA2 and RAD51 and initiates homology-directed DNA repair in a "FA/BRCA pathway', both in response to DNA-damaging agents (cisplatin, ionizing radiation, hydroxyurea, etc.) and in the absence of exogenous DNA damage during the $S$ phase of the cell cycle [24].

Our results revealed an increased protein level of FANCD2, RAD51, BRCA1 and BRIP1 in the NCR compared to $\mathrm{CR}$ cervical tumor nuclei. These observations were also confirmed on an independent validation set, emphasizing the role of these four proteins in CRT resistance (Table 7). Although we observed a 3.8-fold increase in BRCA2 mRNA in NCR vs. CR cervical samples (qRT-PCR data), there was no significant difference for BRCA2 protein between NCR and CR groups, which could be due to either using an inadequate monoclonal antibody clone or posttranscriptional modifications of the BRCA2 transcript. A central step in the FA/BRCA pathway is the monoubiquitylation of FANCD2 and its translocation to chromatin at the site of DNA damage [25]. The ubiquitylation of FANCD2 is initiated by FANCM and is mediated by the UBE2T (E2) enzyme and a multisubunit ubiquitin E3 ligase that consists of eight FA proteins (FANCA/B/C/E/F/G/L/ M) [26]. FANCD2 can also be monoubiquitylated and chromatin-loaded by the E3 ubiquitin ligase activity of RAD18 in a FA-independent manner [27]. 


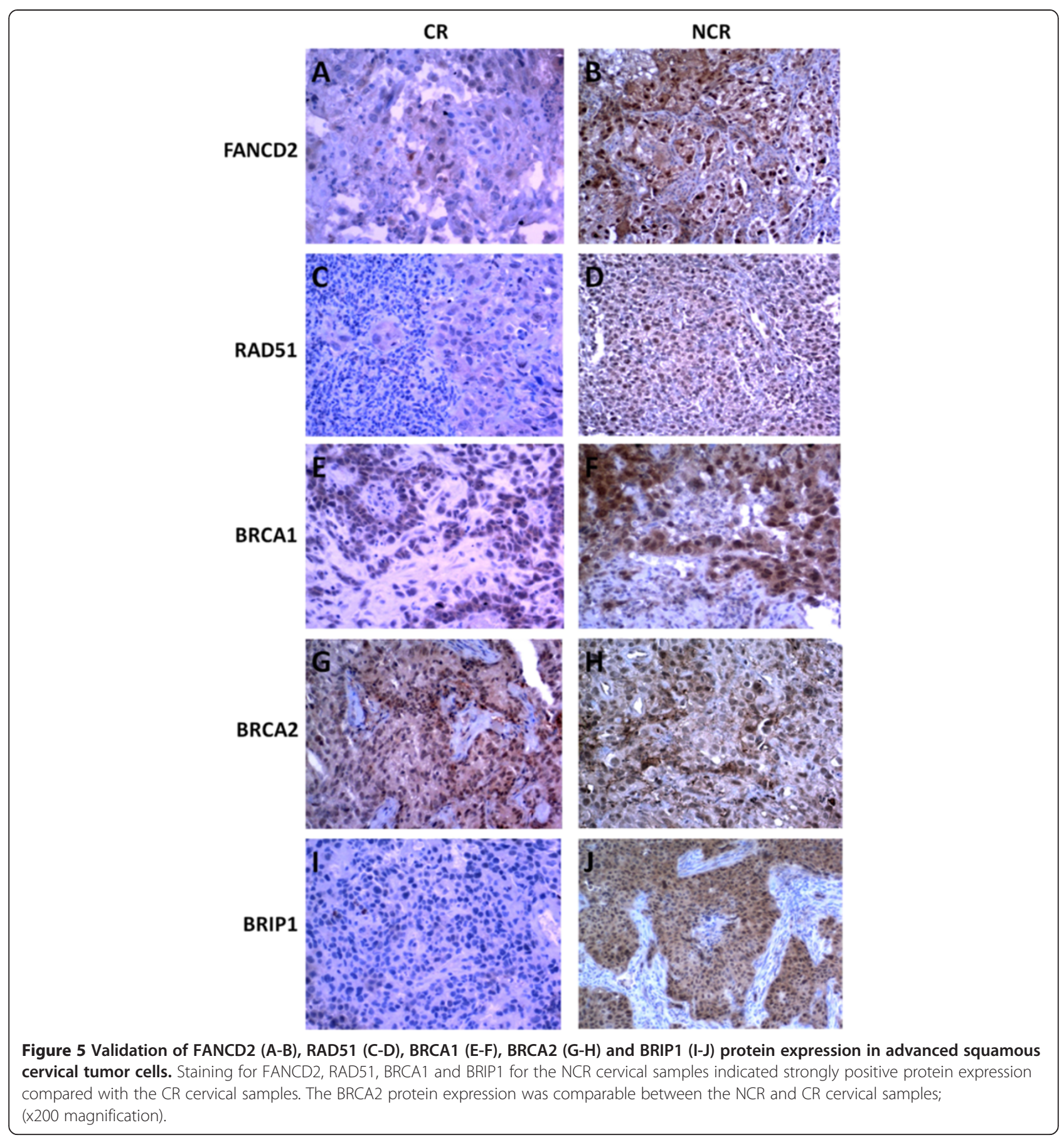

Increased expression of FANCM, FANCL, UBE2T and RAD18 mRNA was observed in NCR compared to CR cervical tumor samples (Additional file 1). We also observed a slight increase in SLX4/FANCP/BTBD12 mRNA (fold change $=1.3, \mathrm{p}=0.035$ ) for the NCR vs. CR cervical samples. SLX4 is a novel member of the FA genes that coordinates multiple DNA repair pathways by acting as a scaffold for multiple nucleases involved in ICL repair and the mechanisms involved in HR [28]. Depletion of SLX4 leads to hypersensitivity to cisplatin and reduced efficiency of HR repair [29]. Narayan et al. [30] showed that advanced cervical cancer is associated with alterations in the FA/ BRCA pathway by either promoter hypermethylation and/ or deregulated gene expression compared with the normal cervix. FA inhibitors were recently proposed as important tools for overcoming cisplatin resistance in tumors [31].

RAD51, one of the key molecules of DNA repair, is another gene we found to be significantly overexpressed in 
Table 7 Assessment of the nuclear proteins including FANCD2, RAD51, BRCA2, BRCA1, BRIP1(BACH1) in NCR and $C R$ cervical tumors evaluated both for genomics study set $(n=21)$ and IHC validation set $(n=24)$

\begin{tabular}{lccccc}
\hline Proteins & $\begin{array}{l}\text { Cellular } \\
\text { localization }\end{array}$ & NCR \% & CR \% & NCR/CR ratio & p-value \\
$\begin{array}{l}\text { Genomics study } \\
\text { set ( } \mathbf{n}=\mathbf{2 1} \text { ) }\end{array}$ & & & & & \\
\hline FANCD2 & nucleus & 32.53 & 15.6 & 2.09 & $\mathbf{0 . 0 3 2}$ \\
\hline RAD51 & nucleus & 14.88 & 7.11 & 2.09 & $\mathbf{0 . 0 1 6}$ \\
\hline BRCA2 & nucleus & 21.11 & 20.81 & 1.01 & 0.868 \\
\hline BRCA1 & nucleus & 27.78 & 10.69 & 2.60 & $\mathbf{0 . 0 3 2}$ \\
\hline BRIP1 (BACH1) & nucleus & 40.44 & 10.05 & 4.02 & $\mathbf{0 . 0 0 1}$ \\
\hline $\begin{array}{l}\text { IHC validation } \\
\text { set (n= 24) }\end{array}$ & & & & & \\
\hline FANCD2 & nucleus & 25.34 & 16.22 & 1.56 & $\mathbf{0 . 0 1 1}$ \\
\hline RAD51 & nucleus & 16.87 & 4.86 & 3.47 & $\mathbf{0 . 0 0 0}$ \\
\hline BRCA1 & nucleus & 27.59 & 12.97 & 2.13 & $\mathbf{0 . 0 0 0}$ \\
\hline BRIP1 (BACH1) & nucleus & 30.73 & 14.55 & 2.11 & $\mathbf{0 . 0 1 1}$ \\
\hline
\end{tabular}

The $p$-values in the bold format are statistically significant.

the NCR cervical cancers. We observed a 3.4-fold increase in RAD51 mRNA (qRT-PCR data) and also an increased protein expression of RAD51 in the nuclei of the NCR vs. CR cervical cancers. RAD51 has anti-apoptotic activity in tumor cells [32], and high expression of this protein is correlated with poor prognosis, resistance to ionizing radiation and drug resistance [33]. RAD51 is essential in the HR process of DNA repair, its expression being tightly controlled in normal healthy cells to maintain genomic stability [34]. RAD51 co-localizes with BRCA1 and FANCD2 in $\mathrm{S}$-phase specific nuclear foci and initiates homology-driven repair activity. Several studies have shown that in cancer cells, this molecule is overexpressed, leading to radio- and chemoresistance [35,36]. Elevated levels of RAD51 have been associated with increased invasiveness in breast cancer patients [37] and have been demonstrated to be an independent prognostic marker of survival in non-small cell lung cancer patients [38]. A previous microarray study of paired cervical tumor samples (pre- and post-chemoradiotherapy) reported down-regulation of RAD51 after treatment [5], supporting the hypothesis that radiation sensitivity is facilitated by a diminished DNA repair response. Targeting strategies against this gene have been developed as possible anticancer treatments; attempts to inhibit RAD51 have proven to be successful in reducing treatment resistance in tumor cells $[39,40]$.

In a previous study, it was shown that poly(ADP-ribose) polymerase (PARP) inhibitors could suppress the expression of BRCA1 and RAD51 [41]. The PARP family, especially PARP1 and PARP2, functions as DNA damage sensors and recruits a variety of DNA repair proteins to the site of damaged DNA [42]. In BRCA-positive breast cancer, PARP inhibitors were found to increase the cytotoxic effects of radiation and chemotherapy based on the principle of synthetic lethality [43]. In our microarray study, we noted a higher level of PARP2 mRNA in the NCR vs. CR cervical cancer samples (Additional file 1).

Previous studies have shown that prognostic factors including younger age [44], tumor size [45], anemia (hemoglobin) and FIGO stage [46], are used to estimate overall survival, disease-free survival and local control in cervical cancer. Nevertheless, they not provide information about the baseline resistance and the tumor heterogeneity. Our data indicates age as significant factor for treatment response, however the sample size is limited and no final conclusions can be drawn. Taking into account our findings and the need to identify new valuable prognostic factors for baseline resistance, we suggest that if our results are confirmed on a larger study, the assessment of nuclear expression of FANCD2, RAD51, BRCA1 and BRIP1 proteins could represent a supplementary prognostic factor that would better tailor the treatment for patients with advanced cervical cancer. These results could be the foundation for the development of new targeting strategies to improve cervical cancer outcome.

\section{Conclusions}

Our data revealed high DNA repair machinery activity even before starting radio-chemotherapy in NCR patients compared with $\mathrm{CR}$ patients. Therefore, our findings demonstrate that baseline FANCD2, RAD51, BRCA1 and BRIP1 nuclear protein expression could have an important role in treatment failure in advanced squamous cervical cancer. To our knowledge, this is the first study to demonstrate the role of the FA/BRCA pathway in baseline resistance and therapy failure in locally advanced cervical cancer.

\section{Limitations}

The limitation of this study is related to the small number of samples even if we used an independent validation set for protein data to increased confidence of these findings. Larger studies have to confirm that the assessment of these proteins could represent an important prognostic factor that determines poor response to radiation and chemotherapy for locally-advanced cervical cancers.

\section{Additional file}

Additional file 1: Genes involved in DNA replication,

recombination, and repair mechanisms.

\section{Abbreviations}

AUC: Area under curve; BT: Brachytherapy; CR: Complete response; CRT: Concomitant chemoradiotherapy; Cy3: Cyanine 3; DAB: Diamino-benzidine tetrachloride; DSBs: DNA double-strand breaks; EBRT: External beam radiotherapy; FDR: False discovery rate; FIGO: International Federation of Gynecology and Obstetrics; HDR: High-dose-rate; HPV: Human papillomavirus; IHC: Immunohistochemistry; IPA: Ingenuity pathway analysis; NCR: Non-complete response; RIN: RNA integrity number; ROC: Receiver operating characteristic. 


\section{Competing interests}

The authors declare that there are no competing interests.

\section{Authors' contributions}

OB designed and coordinated the research study, performed the microarray experiment, interpreted the results and drafted the manuscript; LB performed bioinformatic analysis of microarray data and drafted the manuscript; OT performed qRT-PCR analysis and revised the manuscript; NT performed statistical analysis; MR and SV processed the samples for microarray analysis; RB and SS assessed the IHC staining; BF performed histopathological evaluation of the samples; LP and LM processed the tissues for IHC staining; CO enrolled and followed up patients, IBN interpreted the results and revised the manuscript, VN enrolled and followed up patients and revised the manuscript. All authors read and approved the final manuscript.

\section{Acknowledgements}

The authors acknowledge the financial support provided by the UEFISCDI Program - PN-II-PT-PCCA-2011-3.2-1328 (grant no. 96/2012). Additional grants including the National Grants Program -PNCDI2 (grant no. 42160/2008), the National Grant Program PN-II- ID - PCE- 2008-2, CNCSIS 1532 and PN-II-RU-PD2011-3-0283, supported parts of this work. The authors thank to all of the patients who agreed to enter this study.

\section{Author details}

'The Oncology Institute "Prof Dr. Ion Chiricuta", 34-36 Republicii street, 400015 Cluj-Napoca, Romania. ${ }^{2}$ Iuliu Hatieganu, University of Medicine and Pharmacy, 8 Babes street, 400012 Cluj-Napoca, Romania. ${ }^{3}$ Faculty of Veterinary Medicine, University of Agricultural Sciences and Veterinary Medicine, 3-5 Calea Manastur street, 400372 Cluj-Napoca, Romania.

Received: 12 August 2013 Accepted: 3 April 2014

Published: 8 April 2014

\section{References}

1. Jemal A, Bray F, Center MM, Ferlay J, Ward E, Forman D: Global cancer statistics. CA Cancer J Clin 2011, 61:69-90.

2. Lippert $\mathrm{TH}$, Ruoff HJ, Volm M: Intrinsic and acquired drug resistance in malignant tumors. The main reason for therapeutic failure. Arzneimittelforschung 2008, 58:261-264.

3. American Cancer Society: Cancer Facts \& Figures 2012. Atlanta: American Cancer Society; 2012

4. Branzei D, Foiani M: Regulation of DNA repair throughout the cell cycle. Nat Rev Mol Cell Biol 2008, 9:297-308.

5. Zempolich K, Fuhrman C, Milash B, Flinner R, Greven K, Ryu J, Forbes A, Kerlin K, Nichols RC, Gaffney DK: Changes in gene expression induced by chemoradiation in advanced cervical carcinoma: a microarray study of RTOG C-0128. Gynecol Oncol 2008, 109:275-279.

6. Wong YF, Selvanayagam ZE, Wei N, Porter J, Vittal R, Hu R, Lin Y, Liao J, Shih JW, Cheung TH, Lo KW, Yim SF, Yip SK, Ngong DT, Siu N, Chan LK, Chan CS, Kong T, Kutlina E, McKinnon RD, Denhardt DT, Chin KV, Chung TK: Expression genomics of cervical cancer: molecular classification and prediction of radiotherapy response by DNA microarray. Clin Cancer Res 2003, 9:5486-5492.

7. Chao A, Wang TH, Lai CH: Overview of microarray analysis of gene expression and its applications to cervical cancer investigation. Taiwan $J$ Obstet Gynecol 2007, 46:363-373.

8. Livak KJ, Schmittgen TD: Analysis of relative gene expression data using real-time quantitative PCR and the 2(-Delta Delta $C(T))$ method. Methods 2001, 25:402-408.

9. Tuominen VJ, Ruotoistenmaki S, Viitanen A, Jumppanen M, Isola J: ImmunoRatio: a publicly available web application for quantitative image analysis of estrogen receptor (ER), progesterone receptor (PR), and Ki-67. Breast Cancer Res 2010, 12:R56.

10. Rosner B: Fundamentals of biostatistics. 6th edition. Pacific Grove, CA Duxbury Press; 2006.

11. Zou KH, Liu A, Bandos Al, Ohno-Machado L, Rockette HE: Statistical evaluation of Diagnostic performance topics in ROC Analysis. Boca Raton, Florida: Chapman \& Hall/CRC Press; 2012.

12. Bamber $\mathrm{D}$ : The area above the ordinal dominance graph and the area below the receiver operating characteristics graph. J Math Psychol 1975, 12:387-415.

13. Hanley JA, McNeil BJ: The meaning and use of the area under a receiver operating characteristic (ROC) curve. Radiol 1982, 143:29-36.
14. Kauffmann A, Rosselli F, Lazar V, Winnepenninckx V, Mansuet-Lupo A, Dessen P, van den Oord JJ, Spatz A, Sarasin A: High expression of DNA repair pathways is associated with metastasis in melanoma patients. Oncogene 2008, 27:565-573.

15. Sanchez-Carbayo M, Socci ND, Lozano J, Saint F, Cordon-Cardo C: Defining molecular profiles of poor outcome in patients with invasive bladder cancer using oligonucleotide microarrays. J Clin Oncol 2006, 24:778-789.

16. Rigakos G, Razis E: BRCAness: finding the Achilles heel in ovarian cancer. Oncologist 2012, 17:956-962.

17. Roy R, Chun J, Powell SN: BRCA1 and BRCA2: different roles in a common pathway of genome protection. Nat Rev Cancer 2012, 12:68-78.

18. Zhang Y, Fan S, Meng Q, Ma Y, Katiyar P, Schlegel R, Rosen EM: BRCA1 interaction with human papillomavirus oncoproteins. J Biol Chem 2005, 280:33165-33177.

19. Wysham WZ, Mhawech-Fauceglia P, Li H, Hays L, Syriac S, Skrepnik T, Wright J, Pande N, Hoatlin M, Pejovic T: BRCAness profile of sporadic ovarian cancer predicts disease recurrence. PLoS One 2012, 7:e30042.

20. Wang Y, Cortez D, Yazdi P, Neff N, Elledge SJ, Qin J: BASC, a super complex of BRCA1-associated proteins involved in the recognition and repair of aberrant DNA structures. Genes Dev 2000, 14:927-939.

21. Kerr $P$, Ashworth A: New complexities for BRCA1 and BRCA2. Curr Biol 2001, 11:R668-R676.

22. Jensen RB, Ozes A, Kim T, Estep A, Kowalczykowski SC: BRCA2 is epistatic to the RAD51 paralogs in response to DNA damage. DNA Repair (Amst) 2013, 12:306-311.

23. Kim H, D'Andrea AD: Regulation of DNA cross-link repair by the Fanconi anemia/BRCA pathway. Genes Dev 2012, 26:1393-1408.

24. Wang W: Emergence of a DNA-damage response network consisting of Fanconi anaemia and BRCA proteins. Nat Rev Genet 2007, 8:735-748

25. Alpi AF, Patel KJ: Monoubiquitylation in the Fanconi anemia DNA damage response pathway. DNA Repair (Amst) 2009, 8:430-435.

26. Kelsall IR, Langenick J, MacKay C, Patel KJ, Alpi AF: The Fanconi anaemia components UBE2T and FANCM are functionally linked to nucleotide excision repair. PLoS One 2012, 7:e36970.

27. Williams SA, Longerich S, Sung P, Vaziri C, Kupfer GM: The E3 ubiquitin ligase RAD18 regulates ubiquitylation and chromatin loading of FANCD2 and FANCI. Blood 2011, 117:5078-5087.

28. Svendsen JM, Smogorzewska A, Sowa ME, O'Connell BC, Gygi SP, Elledge SJ, Harper JW: Mammalian BTBD12/SLX4 assembles a Holliday junction resolvase and is required for DNA repair. Cell 2009, 138:63-77.

29. Fekairi S, Scaglione S, Chahwan C, Taylor ER, Tissier A, Coulon S, Dong MQ, Ruse C, Yates JR 3rd, Russell P, Fuchs RP, McGowan CH, Gaillard PH: Human SLX4 is a Holliday junction resolvase subunit that binds multiple DNA repair/recombination endonucleases. Cell 2009, 138:78-89.

30. Narayan G, Arias-Pulido H, Nandula SV, Basso K, Sugirtharaj DD, Vargas H, Mansukhani M, Villella J, Meyer L, Schneider A, Gissmann L, Dürst M, Pothuri B, Murty W: Promoter hypermethylation of FANCF: disruption of Fanconi Anemia-BRCA pathway in cervical cancer. Cancer Res 2004, 64:2994-2997.

31. Jacquemont $C$, Simon JA, D'Andrea AD, Taniguchi T: Non-specific chemical inhibition of the Fanconi anemia pathway sensitizes cancer cells to cisplatin. Mol Cancer 2012, 11:26.

32. Henning W, Sturzbecher HW: Homologous recombination and cell cycle checkpoints: Rad51 in tumour progression and therapy resistance. Toxicology 2003, 193:91-109.

33. Du LQ, Wang Y, Wang H, Cao J, Liu Q, Fan FY: Knockdown of Rad51 expression induces radiation- and chemo-sensitivity in osteosarcoma cells. Med Oncol 2011, 28:1481-1487.

34. Richardson C: RAD51, genomic stability, and tumorigenesis. Cancer Lett 2005, 218:127-139.

35. Tennstedt P, Fresow R, Simon R, Marx A, Terracciano L, Petersen C, Sauter G, Dikomey E, Borgmann K: RAD51 overexpression is a negative prognostic marker for colorectal adenocarcinoma. Int J Cancer 2013, 132:2118-2126

36. Hannay JA, Liu J, Zhu QS, Bolshakov SV, Li L, Pisters PW, Lazar AJ, Yu D, Pollock RE, Lev D: Rad51 overexpression contributes to chemoresistance in human soft tissue sarcoma cells: a role for p53/activator protein 2 transcriptional regulation. Mol Cancer Ther 2007, 6:1650-1660.

37. Barbano R, Copetti M, Perrone G, Pazienza V, Muscarella LA, Balsamo T, Storlazzi CT, Ripoli M, Rinaldi M, Valori VM, Latiano TP, Maiello E, Stanziale P, Carella M, Mangia A, Pellegrini F, Bisceglia M, Muda AO, Altomare V, Murgo R, Fazio VM, Parrella P: High RAD51 mRNA expression characterize 
estrogen receptor-positive/progesteron receptor-negative breast cancer and is associated with patient's outcome. Int I Cancer 2011, 129:536-545.

38. Qiao GB, Wu YL, Yang XN, Zhong WZ, Xie D, Guan XY, Fischer D, Kolberg HC, Kruger S, Stuerzbecher HW: High-level expression of Rad51 is an independent prognostic marker of survival in non-small-cell lung cancer patients. Br J Cancer 2005, 93:137-143.

39. Russell JS, Brady K, Burgan WE, Cerra MA, Oswald KA, Camphausen K, Tofilon PJ: Gleevec-mediated inhibition of Rad51 expression and enhancement of tumor cell radiosensitivity. Cancer Res 2003, 63:7377-7383.

40. Hine CM, Seluanov A, Gorbunova V: Use of the Rad51 promoter for targeted anti-cancer therapy. Proc Natl Acad Sci U S A 2008, 105:20810-20815.

41. Yelamos J, Schreiber V, Dantzer F: Toward specific functions of poly(ADPribose) polymerase-2. Trends Mol Med 2008, 14:169-178

42. Wang $X$, Weaver DT: The ups and downs of DNA repair biomarkers for PARP inhibitor therapies. Am J Cancer Res 2011, 1:301-327.

43. Sandhu SK, Yap TA, de Bono JS: The emerging role of poly (ADP-Ribose) polymerase inhibitors in cancer treatment. Curr Drug Targets 2011, 12:2034-2044

44. Fyles AW, Pintilie M, Kirkbride P, Levin W, Manchul LA, Rawlings GA: Prognostic factors in patients with cervix cancer treated by radiation therapy: results of a multiple regression analysis. Radiother Oncol 1995, 35:107-117.

45. Narayan K, Fisher R, Bernshaw D: Significance of tumor volume and corpus uteri invasion in cervical cancer patients treated by radiotherapy. Int J Gynecol Cancer 2006, 16:623-630.

46. Grigiene R, Valuckas KP, Aleknavicius E, Kurtinaitis J, Letautiene SR: The value of prognostic factors for uterine cervical cancer patients treated with irradiation alone. BMC Cancer 2007, 7:234.

doi:10.1186/1471-2407-14-246

Cite this article as: Balacescu et al.: Gene expression profiling reveals activation of the FA/BRCA pathway in advanced squamous cervical cancer with intrinsic resistance and therapy failure. BMC Cancer 2014 14:246

\section{Submit your next manuscript to BioMed Central and take full advantage of:}

- Convenient online submission

- Thorough peer review

- No space constraints or color figure charges

- Immediate publication on acceptance

- Inclusion in PubMed, CAS, Scopus and Google Scholar

- Research which is freely available for redistribution 